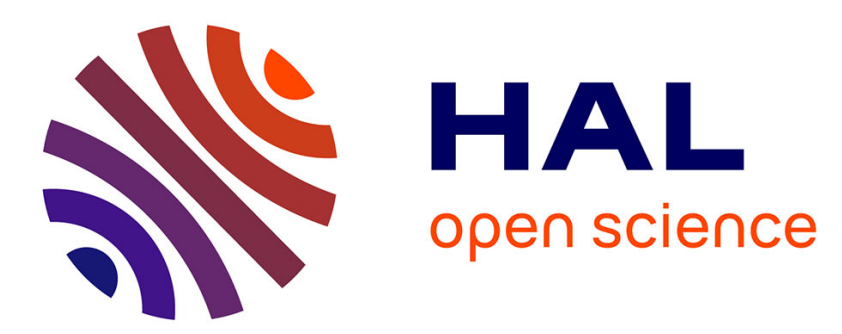

\title{
Determination of hemolysis cut-offs for biochemical and immunochemical analytes according to their value.
} Anne Marie Dupuy, Anne-Sophie Bargnoux, Nils Kuster, Jean-Paul Cristol, Stéphanie Badiou

\section{To cite this version:}

Anne Marie Dupuy, Anne-Sophie Bargnoux, Nils Kuster, Jean-Paul Cristol, Stéphanie Badiou. Determination of hemolysis cut-offs for biochemical and immunochemical analytes according to their value.. Clinical Chemistry and Laboratory Medicine, 2020, 10.1515/cclm-2019-1228 . hal-02538430

\author{
HAL Id: hal-02538430 \\ https://hal.science/hal-02538430
}

Submitted on 2 Oct 2021

HAL is a multi-disciplinary open access archive for the deposit and dissemination of scientific research documents, whether they are published or not. The documents may come from teaching and research institutions in France or abroad, or from public or private research centers.
L'archive ouverte pluridisciplinaire HAL, est destinée au dépôt et à la diffusion de documents scientifiques de niveau recherche, publiés ou non, émanant des établissements d'enseignement et de recherche français ou étrangers, des laboratoires publics ou privés. 


\section{Determination of hemolysis cut-offs for biochemical and immunochemical analytes according to their value}

\section{Abstract}

Background: All general biochemistry instruments allow the measure of hemolysis index (HI), and suppliers provide an acceptable $\mathrm{HI}$ for each assay without consideration of the analyte value or its clinical application. Our first objective was to measure the impact of hemolysis degree on plasma biochemical and immunochemical analytes to determine the maximum allowable HI for each of them using four calculation methods as significant bias in comparison to manufacturer's data. The second objective was to assess whether the maximum allowable $\mathrm{HI}$ varied according to the analyte values.

Methods: Twenty analytes were measured in hemolyzatetreated plasma to determine the HI leading to a significant change compared to baseline value. Analytes were assessed at one ( 3 analytes), two (5 analytes) and three (12 analytes) values according to their sensitivity to hemolysis and their clinical impact. We used four calculation methods as significant limit from baseline value: the total change limit (TCL), the $10 \%$ change $(10 \% \Delta)$, the analytical change limit and the reference change value.

Results: Allowable HI was significantly different according to the threshold chosen for most analytes and was also dependent on the analyte value for alkaline phosphatase, alanine aminotransferase, aspartate aminotransferase, creatine kinase, iron, haptoglobin and high sensitivity troponin T. No hemolysis interference was observed for

*Corresponding author: Jean Paul Cristol, Department of Biochemistry, Lapeyronie University Hospital, 191 Avenue du Doyen Gaston Giraud, 34295 Montpellier Cedex 5, France; and PhyMedExp, INSERM, CNRS, University of Montpellier, Montpellier, France, Fax: +334673383 93, E-mail: jp-cristol@chu-montpellier.fr Anne Marie Dupuy: Department of Biochemistry, Lapeyronie University Hospital, Montpellier, France

Anne Sophie Bargnoux, Nils Kuster and Stéphanie Badiou: Department of Biochemistry, Lapeyronie University Hospital, Montpellier, France; and PhyMedExp, INSERM, CNRS, University of Montpellier, Montpellier, France albumin, creatinine, C-reactive protein, and procalcitonin even at an $\mathrm{HI}$ value of $11 \mathrm{~g} / \mathrm{L}$.

Conclusions: This study highlights that TCL is the most appropriate calculation method to determine allowable $\mathrm{HI}$ in practice for biochemical and immunochemical parameters using Cobas 8000@ from Roche Diagnostics. In addition, different allowable HI were found according to analyte value leading to optimization of resampling to save time in patient care.

Keywords: analytes; hemolysis index; interference; total change limit; validity of results.

\section{Introduction}

High sample quality is essential to ensure the reliability of results for appropriate patient care. The laboratories must manage the entire analytical process from pre- to postanalytical stages. Among the many indicators of sample quality, hemolysis is a major issue in laboratory medicine which can lead to sample rejection, resampling and delay in the laboratory result [1]. Hemolysis is more frequently observed in emergency department (ED) samples than in other medical units [2, 3]. In our laboratory, based on the results of 13,351 consecutive samples, we determined that up to $4.0 \%$ vs. $<1.0 \%$ of samples from ED vs all other medical units, respectively, had an $\mathrm{HI}>3.0 \mathrm{~g} / \mathrm{L}$. A recent survey reported an overall hemolysis rate of $3.4 \%$ with a higher occurrence in samples from inpatients compared to outpatients [4]. The possible solutions to reduce hemolysis rates in the ED or ICU could be the implementation of low vacuum tubes in the use of IV catheter blood collection [5]. The management of hemolysis varies according to the institution and the methods of determining interference. Hemolyzed samples can have detrimental consequences on the quality of patient care.

For example, great hemolysis can lead to higher potassium or lower troponin $\mathrm{T}$ levels, which can delay diagnosis and patient care [4]. These consequences, along with the repetition of analysis, can also cause an increase in cost. 
Currently, only two manufacturers (Roche and Abbott) offer an automated measurement of the hemolysis index (HI) which can be converted into the corresponding hemoglobin value in $\mathrm{g} / \mathrm{L}$, along with icterus and lipemia indices. However, the $\mathrm{HI}$ associated to a significant interference for each assay and provided by manufacturers has been determined using a classical 10\% change [6] from baseline value $(10 \% \Delta)$ within reference interval, while the impact of interference likely depends on the value of the analytes. For this reason, manufacturer's data on allowable $\mathrm{HI}$ are frequently over- or underestimated [7]. To overcome this, the Clinical and Laboratory Standards Institute (CLSI) recommends measuring the index at a minimum of two medical decision values to evaluate interference [8]. In addition to analytical and biological criteria, a clinical basis should be considered due to the clinical impact of hemolysis interference. The CLSI guideline states that an assay's instruction for use (IFU) should document the values of both hemoglobin and analyte that interfere as well as the amount of bias observed [9]. However, to date, IFUs are not fully compliant with CLSI guidelines. In consequence, laboratories cannot simply assume that manufacturers are performing testing at medically relevant decision levels, and the information contained within IFUs may be inadequate. So, it appears useful to assess hemolysis interference at several values of a given analyte in order to avoid erroneous results and to limit the collection of additional samples. Moreover, the maximum allowable interference resulting in the bias of a measured analyte could be dependent on the choice of calculation method which must be carefully considered [10]. Beyond the classical $10 \% \Delta$ threshold, other calculation methods of interest are based on the analytical change limit (ACL), the total change limit (TCL) or the reference change value (RCV) [11]. The ACL, based on analytical variation and often used for stability assessment, enables to define whether the observed variation is statistically significant. However, it is more stringent than $10 \% \Delta$ and too restrictive according to different authors [10, 12]. The TCL is widely used to assess interferences or stability of biochemical analytes in biological fluid, and it takes both analytical and biological variations into account [13]. RCV, which also accounts for both analytical and biological variations, is currently designed for the interpretation of serial measurements but is not restrictive enough for some analytes such as troponin [10]. Clearly, biological variation is important; however, regarding some analytes for which data on biological variation do not exist, the ACL calculation method may represent here an alternative solution.

The first objective of our study was to measure the impact of hemolysis degree on plasma biochemical and immunochemical analytes to determine the maximum allowable $\mathrm{HI}$ for each of them using four calculation methods as significant bias from baseline value (TCLbased HI cut-off, 10\% 0 -based HI cut-off, ACL-based HI cut-off and RCV-based HI cut-off). Then, our own HI cut-off limits were compared to manufacturer's data. The second objective was to assess whether the maximum allowable HI varied according to the analyte value. Three levels of values (upper limit, lower limit and within reference interval) were selected for 12 analytes known to be sensitive to hemolysis [1]. Two levels of values for five analytes and one for three analytes were selected guided by clinical application to confirm and/or complete literature's data $[6,8]$.

\section{Materials and methods}

\section{Study design}

Twenty routine biochemical and immunochemical analytes were measured on heparinized plasma pools free of hemoglobin (baseline value) and after overload cascade with hemoglobin-titrated hemolyzate. Measurements were performed on a Cobas ${ }^{\circledR} 8000$ analyzer (Roche diagnostics, Meylan, France), which has the ability to assess HI which is then converted into hemoglobin value. We considered as allowable $\mathrm{HI}$ the highest $\mathrm{HI}$ measurement without significant change of analyte value from baseline. After calculating the bias (overloaded sample value - initial value) we compared this value with the different interference criteria such as $10 \% \Delta$, ACL, TCL or RCV. When the bias was higher than interference criterion, the change was considered as significant. This process was approved by the local Ethics Committee (Lapeyronie University Hospital, Montpellier, France).

\section{Analytes investigated and $\mathrm{HI}$ measurement}

We focused on analytes with low HI, with discordant data in the literature, with discordant results compared to Roche's HI, or not studied by other authors. Three values of baseline value were tested for the 12 following analytes: alkaline phosphatase (ALP), alanine aminotransferase (ALT), aspartate aminotransferase (AST), bilirubin (direct), creatine kinase (CK), $\gamma$-glutamyltransferase (GGT), haptoglobin (Hp), high sensitivity troponin T (hs-cTnT), iron, lactate dehydrogenase (LD), phosphorus and potassium. Five additional analytes were studied at two levels of values: albumin, creatinine, ferritin, lipase and protein. Three additional analytes known to be less influenced by hemolysis or widely studied were assessed at only one value: bicarbonates, C-reactive protein (CRP) and procalcitonin (PCT). Values were chosen to be either in the reference interval of lab or close to clinical decision values, or in pathological range. All measurements were performed in duplicate on a Kryptor CompactPlus@ (ThermoFisher, Villebon Sur Yvette, France) for PCT and on a Cobas ${ }^{\circledR} 8000$ Analyzer (Roche Diagnostics, Meylan, France) for the 19 other analytes. The Cobas ${ }^{\circledR} 8000$ is formed by an ion selective 


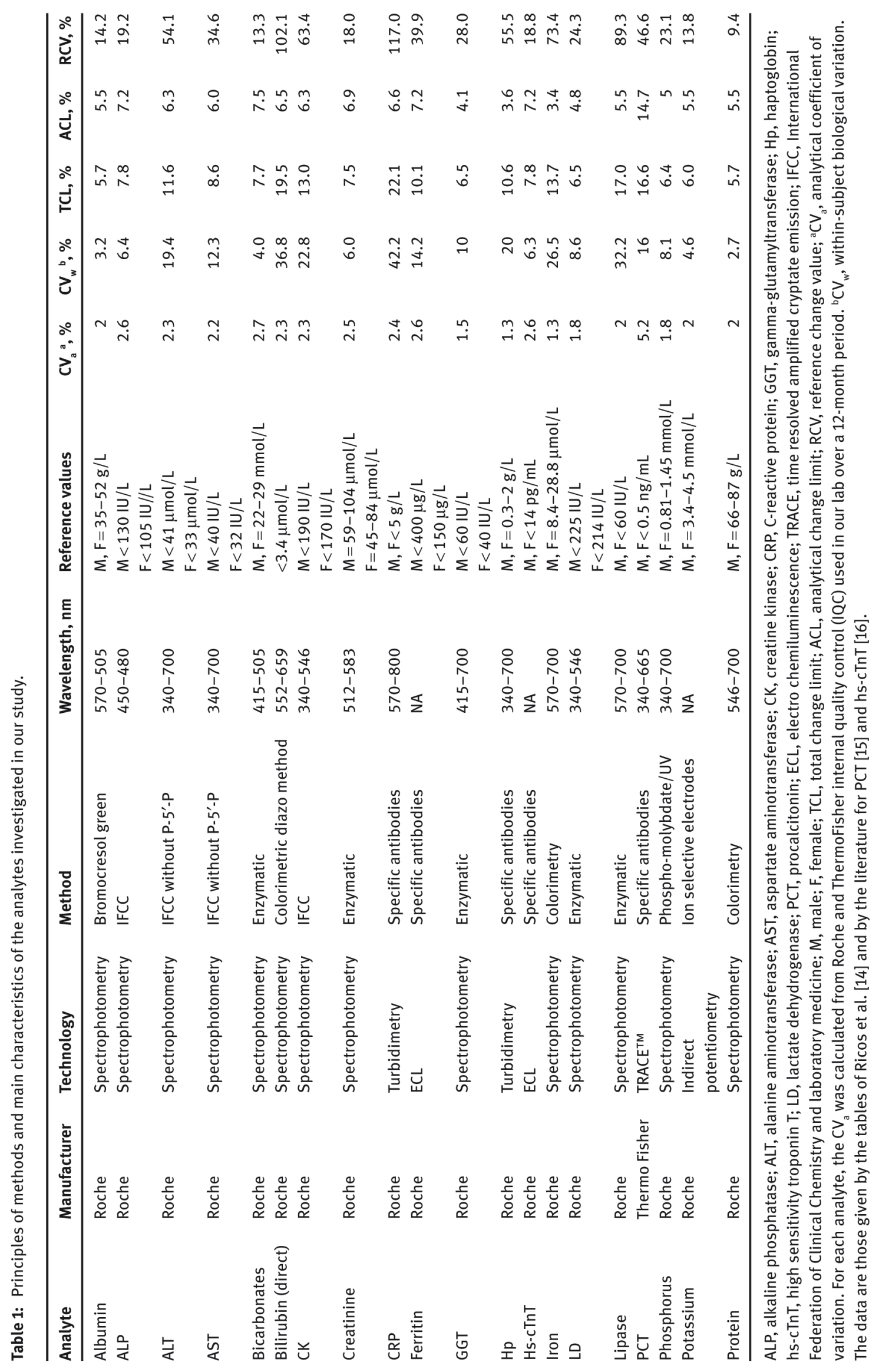


electrode module, c701 and c502 modules (for photometric assays) and an e602 module (for immunoassays). HI was measured on the c701 module by a bichromatic wavelength-paired measurement at 570 and $600 \mathrm{~nm}$. The final result of HI was expressed as an absolute number which corresponds approximately to the hemoglobin value in $\mathrm{g} / \mathrm{L}$ (measuring range $0.05-11 \mathrm{~g} / \mathrm{L}$ ). The principles of methods and the main characteristics of the analytes investigated are reported in Table 1.

\section{Preparation of samples}

The preparation of samples followed three phases: phase 1, preparation of hemolyzate; phase 2, selection of hemoglobin-free samples to form a heparinized plasma pool with analytes of value of interest; and phase 3, overload of each pool of plasma with hemolyzate. Hemolyzate was prepared according to the protocol CLSI EP7-A2 using the osmotic shock procedure [8]. After removing plasma from heparinized whole blood pool of 10 samples, the red blood cells were washed three times in a $0.9 \% \mathrm{NaCl}$ solution and then lysed in distilled water and frozen overnight at $-20^{\circ} \mathrm{C}$. After thawing, hemoglobin value in the hemolyzate was determined and adjusted with distilled water.

We selected from routine plasma free of hemoglobin (with $\mathrm{HI}<0.05 \mathrm{~g} / \mathrm{L}$ ) near the test value desired, and we prepared plasma pools at different values for each analyte.

Each pool of plasma free of hemoglobin $(<0.05 \mathrm{~g} / \mathrm{L})$ was split in equal volumes to be overloaded with increasing amounts of hemoglobin-titrated hemolyzate under a constant total volume of tube of $1 \mathrm{~mL}$ with compensation by addition of $\mathrm{NaCl}$ to obtain the theoretical final values of hemoglobin ranging from 0.1 to $11 \mathrm{~g} / \mathrm{L}$.

\section{Allowable $\mathrm{HI}$ determination}

The HI and the value of analytes were measured in duplicate on Cobas 8000 and Kryptor on each overloaded plasma.

The formula for calculating the hemolysis index on the Cobas8000 is as follows:

$$
\mathrm{H}=1 / \mathrm{A} \cdot\left(\mathrm{Abs}_{2}-\mathrm{B} \cdot \mathrm{Abs}_{1}\right)
$$

with $\mathrm{H}=$ serum indices for hemolysis; $\mathrm{A}$ =factor for conversion of absorbance values $\left(\times 10^{4}\right)$ to serum indices; $\mathrm{Abs}_{2}=$ bichromatic absorbance readings at 505 and $480 \mathrm{~nm}$ for hemolysis; $\mathrm{Abs}_{1}=$ bichromatic absorbance readings at 700 and $660 \mathrm{~nm}$ for lipemia; and $\mathrm{B}=$ corrects hemoglobin measurement $\mathrm{Abs}_{2}$ for lipemia.

For each analyte, the bias was determined as the difference between average of baseline values determined in pools free of hemolysis $\left(\mathrm{T}_{0}\right)$ and average of values obtained from each overload of pools $\left(T_{x}\right)$. The percentage deviation from $T_{0}$ was calculated using Eq. (1):

$$
\left[\frac{\mathrm{T}_{\mathrm{x}}-\mathrm{T}_{0}}{\mathrm{~T}_{0}}\right] \times 100
$$

Each calculated bias was compared to the four cut-offs (10\% 0 , ACL, TCL or RCV), for all analytes.

The TCL, as described by Oddoze et al., was calculated using Eq. (2) [13]:

$$
\left[\left(2.77 \times \mathrm{CV}_{\mathrm{a}}\right)^{2}\right]^{1 / 2}+\left[\left(0.5+\mathrm{CV}_{\mathrm{w}}\right)^{2}\right]^{1 / 2}
$$

where $\mathrm{CV}_{\mathrm{a}}$ is analytical imprecision and $\mathrm{CV}_{\mathrm{w}}$ is within-subject biological variation.

The ACL was calculated using Eq. (3) [17]:

$$
2.77 \times \mathrm{CV}_{\mathrm{a}}
$$

The RCV was calculated according to Eq. (4) [9]:

$$
2.77 \times\left(\mathrm{CV}_{\mathrm{a}}^{2}+\mathrm{CV}_{\mathrm{w}}^{2}\right)^{1 / 2}
$$

For each analyte, the $\mathrm{CV}_{\mathrm{a}}$ (Table 1) was calculated from Roche and ThermoFisher internal quality control (IQC) used in our lab over a 12-month period. The lot number of IQC from Roche was PreciControl ClinChem Multi-160407 for chemistry analysis, PreciControl Varia-276843 for ferritin, PreciControl Cardiac-175878 for myoglobin and NT-proBNP and PreciControl Troponin-195257 for hs-cTnT. The lot number was 25032A for PCT on Kryptor.

The $\mathrm{CV}_{\mathrm{w}}$ are the specifications (2014) provided by Ricos et al. [14] and by the literature for PCT [15] and hs-cTnT [16] (see Tables 1 and 2).

The allowable HI for each analyte was defined as the last $\mathrm{HI}$ value without bias higher than cut-off criteria, using either TCL, $10 \% \Delta$, ACL or RCV as significant limit of bias. Effectively, when the bias (in percentage) was higher than TCL, $10 \% \Delta$ ACL or RCV values, the variation was considered as significant.

\section{Results}

Results of allowable $\mathrm{HI}$ determined for each analyte according to the four calculation methods (TCL, $10 \% \Delta$, ACL, RCV) as well as data provided by the manufacturers are reported in Table 2. Whatever the calculation methods used, there was no hemolysis interference on albumin, creatinine, CRP and PCT even when the HI values were as high as $11 \mathrm{~g} / \mathrm{L}$. Hemolysis led to a positive bias for ALT, AST, CK, iron, LD, lipase, phosphorus, potassium and protein measurement, while a negative bias was observed for ALP, bicarbonates, direct bilirubin, Hp and hs-cTnT.

For ALP, ALT, bilirubin, CK, GGT, iron and lipase, the allowable HI was found higher using TCL or RCV compared to the $10 \% \Delta$-based $\mathrm{HI}$, and these resulted in higher allowable HI than with the ACL-based HI. The lowest HI were obtained for AST, direct bilirubin and LD. Nevertheless, for CK, iron, phosphorus and lipase the HI claimed by Roche were higher than our own measured HI using the $10 \% \Delta$-based HI. All other analytes were less affected by hemolysis than the manufacturer's claim. Ferritin, iron, Hp and GGT showed the highest difference between the allowable HI we determined and those provided by Roche (Table 2). To the best of our knowledge, such study including PCT and ferritin have not been previously investigated in the literature.

Allowable HI was found to be value-dependent for most analytes especially for ALP, ALT, AST, CK, ferritin, 


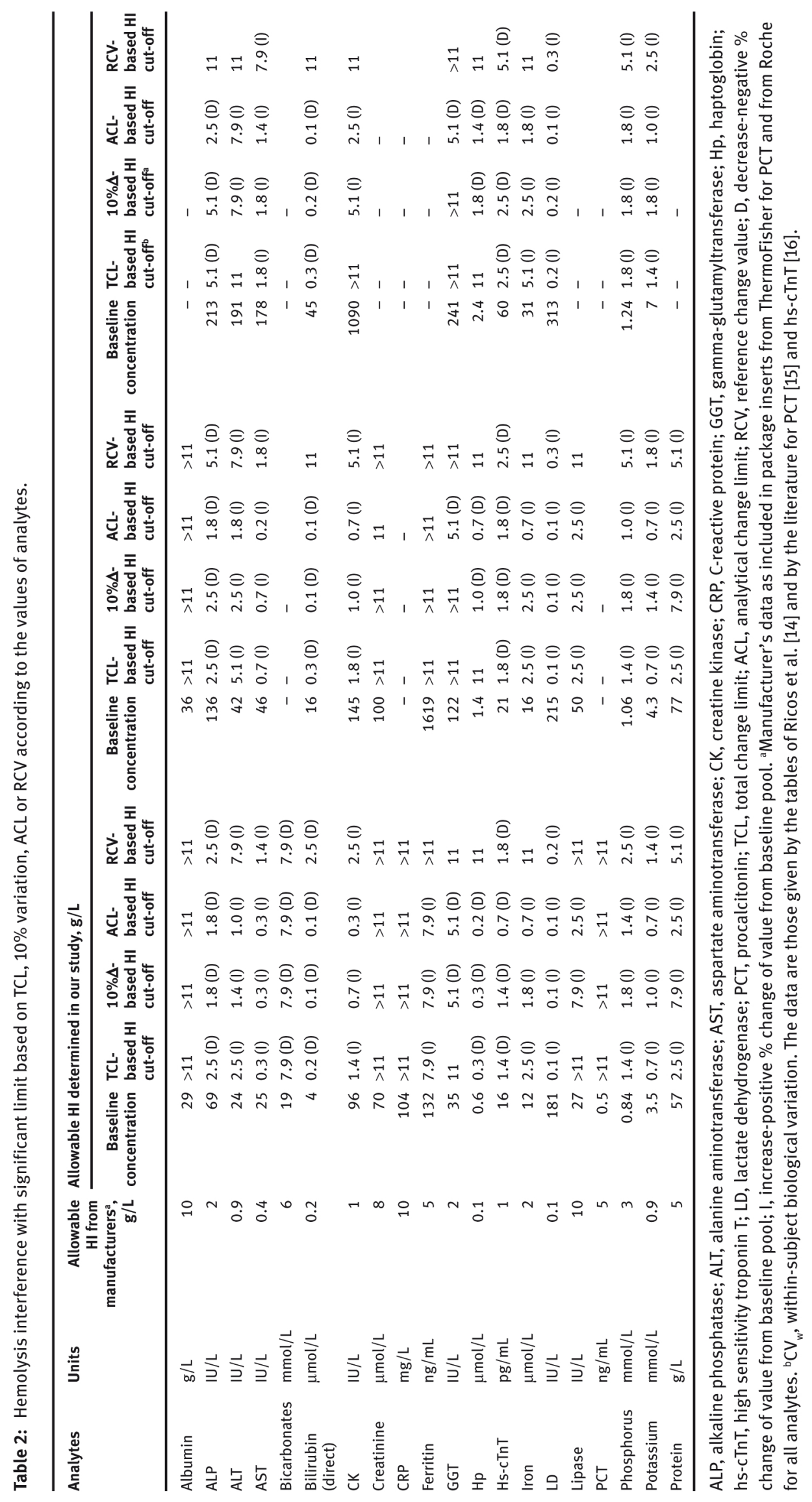



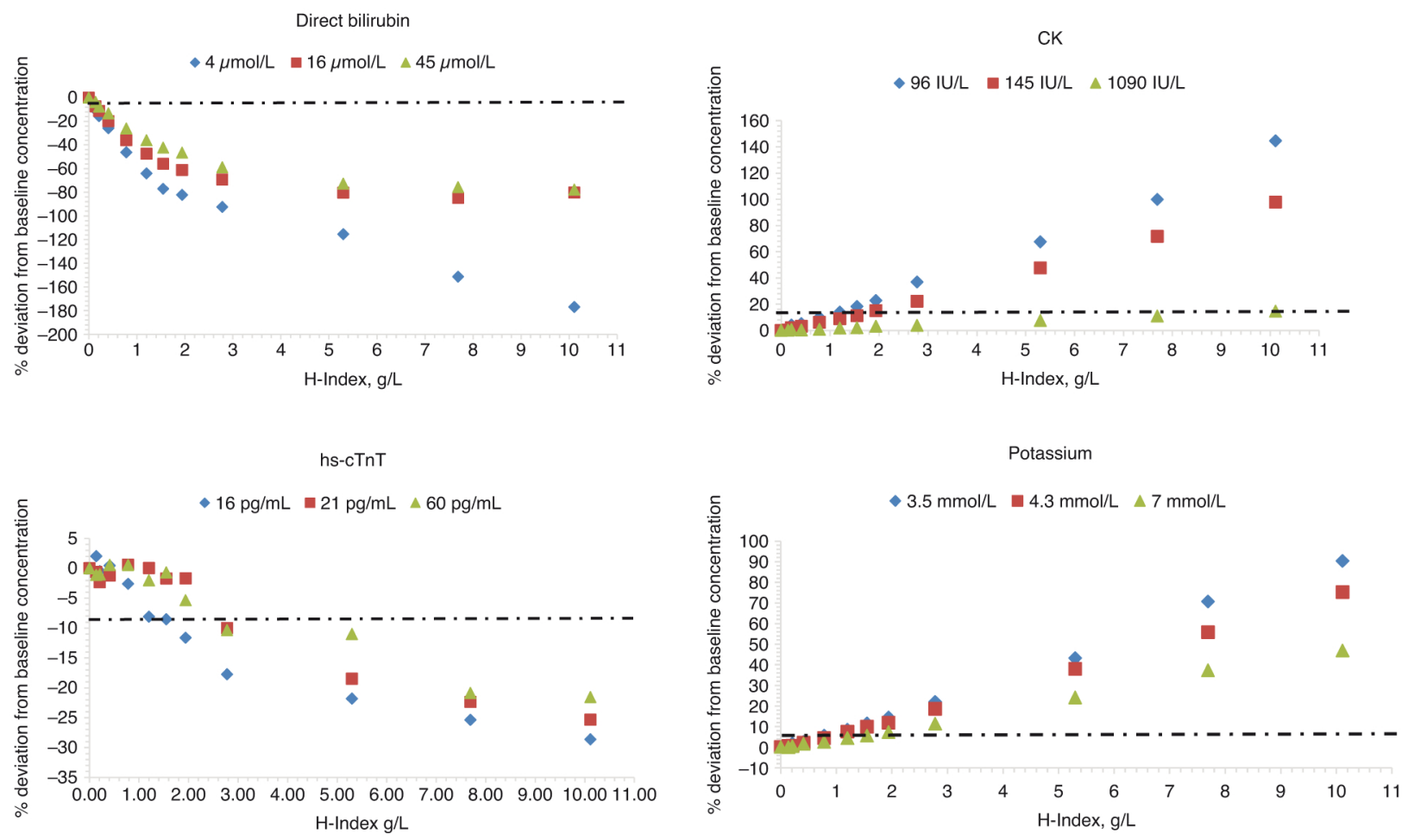

Figure 1: Graphs demonstrating the interference due to hemolysis at different values of analytes tested.

Considering the TCL as maximum percentage acceptable limit, graphs represent the percentage deviation from baseline value of direct bilirubin (A), CK (B), hs-cTnT (C) and potassium (D) according to the hemolysis index. Horizontal dotted line indicates TCL value for each analyte in \%, established from our data. CK, creatine kinase; Hp, haptoglobin; hs-cTnT, high sensitivity troponin T; HI, hemolysis index; $\mathrm{TCL}$, total change limit.

Hp, Hs-cTnT, lipase and potassium. Two schemes were observed: either a linear relationship between the increases in analyte value and $\mathrm{HI}$ (e.g. CK, Hp, hs-cTnT and potassium) or an inverse relationship (e.g. lipase). As seen in Figure 1, the effect of hemolysis for direct bilirubin and hs-cTnT led to a negative bias with increasing amount of hemolyzate and at all analyte values. For potassium, the bias was positive with increasing hemolysis. Interference from hemolysis was significant for the lowest CK value but negligible for the highest. Interferographs of other analytes with percentage changes of analyte values according to the degree of hemolysis are represented in Figure A (Supplementary material).

\section{Discussion}

Our data indicate that some hemolysis index values are not fully concordant with those provided by the manufacturers or published in the literature. In addition, this study clearly demonstrates that the impact of hemolysis depends on analyte values for most analytes tested.
The effect of hemolysis on the analytes studied is in agreement with previous studies $[10,18]$ such as negative biases observed for hs-cTnT and positive biases for others such as potassium. As expected, the more sensitive analytes to hemolysis were AST, direct bilirubin, Hp and LD in agreement with published data [19]. No hemolysis interference was observed for albumin, creatinine and CRP even at an $\mathrm{HI}$ value of $11 \mathrm{~g} / \mathrm{L}$, which is in line with previous reports $[7,18]$. Our data extend the lack of interference to hemolysis (HI $>11 \mathrm{~g} / \mathrm{L}$ ) for high values of ferritin and decisional values of PCT whatever the thresholds used. This could contribute to an appropriate interpretation of data in the event of such a level of hemolysis and could thus avoid resampling.

Hemolysis may interfere with chemical and immunochemical analytes through several mechanisms [9]. Due to the rich cell content in ALT, AST, LD, phosphate and potassium, lysis results in a release of these analytes in plasma, leading to an increase in their plasma value, even at a microscopic level of hemolysis. This is particularly difficult for potassium interpretation, especially in the case of a patient with hypokalemia for whom the interpretation may be erroneous by the presence of hemolysis. 
The additive effect of hemoglobin on total protein value is small but still significant. Dilution is another possible effect especially for gross hemolysis and may result in decreased values. This is typically the case for albumin. At 417, 540 and $575 \mathrm{~nm}$, wavelengths at which hemoglobin strongly absorbs light, hemolysis results in an increase in absorption and a subsequent apparent increase in analyte values measured at these wavelengths. Spectral overlap and chemical reaction between hemolyzate and reaction components represent the cause of hemolysis interference for iron, creatinine and GGT. Regarding ALP, the interference is due to a denaturation of hemoglobin during reaction leading a decrease in ALP values. Increased values of bilirubin even with a slight hemolysis are explained by the pseudo-peroxidase activity of free hemoglobin in inhibiting diazonium color formation. CK is not a constituent of erythrocyte, but its increase during hemolysis is due to the release of active intracellular adenyl cyclase.

In our lab, the measurement of $\mathrm{HI}$ is performed on all plasma/serum tubes on Cobas 8000 through a programming of the data manager in parallel with analyte measurement and is reported for all patient records in the laboratory information system. In view of our experience, it is clear that the automated hemolysis index is of great interest for the monitoring and management of patients (in particular, those from ICU and ED). Several approaches within our lab have been implemented including the use of partial draw tubes with reduced vacuum (BD Vacutainer ${ }^{\circledR}$ Barricor tube) for ED to reduce hemolyzed samples. In addition, HI is recorded in the laboratory information system. An algorithm has been established taking into account the analyte and its value and the value of $\mathrm{HI}$ to either trigger an alert on the presence of hemolysis and add a specific comment under the result by specifying the direction of variation of the result or to remove the result.

If most clinicians are aware of the potential for overestimation due to hemolysis, they are less sensitive about negative interference, for example, on troponin T, underlining the need for laboratories to implement actions for hemolyzed samples. For this, there are two main challenges which are the speed of hemolysis detection and the definition of acceptability criteria to assess the interference of hemolysis in order to cancel the results or report a comment. The establishment of thresholds should be discussed with clinicians, to appreciate under what circumstances the results should be suppressed or not depending on the clinical context. For example, low potassium values in the presence of hemolysis can be a concealed severe hypokalemia. In this context, maintaining the result on the report should be mandatory to alert clinicians.
Today, there is a lack of harmonization for automated HI measurements and for the actions to be taken following the report of results [20]. Allowable HI can be used as supplied by the manufacturers or after own determination. The reasons for the difference between Roche's data and ours could be the method of obtaining the interferent (hemolyzate/Hb overload), the value of analyte studied (no information in the technical sheet on the value of analyte tested) and the cut-off used different from ours. As previously reported [10] and illustrated in the present study, the influence of hemolysis strongly depends on the calculation method used to define the significant bias. Allowable HI was systematically lower when using ACL-based HI cut-off - which considers analytical variation only, compared to the TCL- or RCV-based HI cut-offs [10]. Thus, the clinical impact of the analyte measurement should be taken into account to define a significant bias. For troponin, determining a TCL-based HI cut-off (9.4\%) or a $10 \% \Delta$-based HI cut-off seems more appropriate than using a RCV-based HI cut-off (which was close to 20\%) in line with the European Society of Cardiology (ESC) recommendations for troponin kinetic interpretation [21].

As illustrated in the present study, using own determination of TCL-based HI cut-off would be more appropriate for clinical application than $10 \% \Delta$ as a significant limit of bias. This process could reduce the number of invalid results and the useless need for resampling as well as faster patient management, especially in the ED. This is of particular interest for PCT with an allowable HI value at $5 \mathrm{~g} / \mathrm{L}$ provided by the manufacturers, while our results did not highlight any significant variation up to $11 \mathrm{~g} / \mathrm{L}$ around the cut-off value of $0.5 \mathrm{ng} / \mathrm{mL}$.

Moreover, the HI provided by manufacturers was mainly established using one analyte value within the physiological range. However, the CLSI guidelines [8] recommend that hemolysis interference evaluation should be carried out at a minimum of two values of the analyte. Based on these data and the clinical application of the analytes, we assessed two or three values for 17 analytes. For all of them, except proteins and LD, hemolysis interference was dependent on their value. Surprisingly, for lipase the allowable HI value decreased with a slight increase in the baseline value. For other analytes, we observed that the allowable HI value was higher when the baseline analyte value was high even for an analyte known to be very sensitive to hemolysis like Hp. Indeed, using the TCL-based HI cut-off, the allowable HI value was $0.8 \mathrm{~g} / \mathrm{L}$ for an $\mathrm{Hp}$ value of $0.6 \mathrm{~g} / \mathrm{L}$ compared to a HI value of $11 \mathrm{~g} / \mathrm{L}$ for a value in Hp higher than $1.4 \mathrm{~g} / \mathrm{L}$. Likewise, the allowable HI value for baseline hs-cTnT around the upper limit of 99th percentile value of $14 \mathrm{ng} / \mathrm{L}$ was 
$1.4 \mathrm{~g} / \mathrm{L}$ compared to the $\mathrm{HI}$ value at $2.5 \mathrm{~g} / \mathrm{L}$ if the troponin value was higher than $50 \mathrm{ng} / \mathrm{L}$. High caution should be taken when troponin results are reported in the presence of hemolysis as a significant negative bias could compromise the diagnosis of myocardial infarction based on serial change of troponin value $>20 \%$ according to the ESC [21]. Our results are consistent with previous data which reported an effect of hemolysis on value-dependent troponin $T[7,22]$. Both of these studies reported a negative interference with increasing degrees of hemolysis for the hs-cTnT assay. However, Florkowski et al. [22] observed no interference regardless of the degree of hemolysis on the pool at $<3 \mathrm{ng} / \mathrm{L}$ and a bias $>10 \%$ for the other three pools (pools at 19, 87 and $273 \mathrm{ng} / \mathrm{L}$ ) when the HI was close to $1.32 \mathrm{~g} / \mathrm{L}$. To illustrate the complexity for clinicians, this previous study reported an opposite effect of hemolysis on troponin I value for some assays but not all [22]. The Abbott cTnI assay showed no interference with increasing degrees of hemolysis in a low pool, whereas with the Vitros cTnI assay, the authors reported a positive interference (up to $576 \%$ in low pool). The mechanisms by which hemolysis interferes with the immunoassays appear to be multifactorial and specific to the assay [23]. Indeed, hemolysis would release proteases that cleave the antigenic region of the hs-cTnT recognized in the Roche hs-cTnT immunoassay. On the other hand, the increase in analyte value with hemolysis for Vitros cTnI could be due to the fact that hemolysis at low analyte values causes a weak affinity binding of an erythrocyte component to unoccupied capture antibodies [23].

\section{Limitations of the study}

We determined the impact of hemolysis in our study on an experimental hemolysis, which is different from natural hemolysis. The study of Dimeski et al. [24] pointed out the importance of the strategies to evaluate the impact of hemolysis on the Roche ammonia. Adding hemolyzate to the samples had a dramatic effect on the ammonia results. In the hemolyzate the deamidation of cellular proteins causes an increase in ammonia. However, until now the effect of hemolysis was often studied by adding hemolyzate to plasma/serum based on CLSI. In a recent study [25], no difference was observed on biochemistry results regardless of the method (addition of hemolyzate or naturally hemolyzed patient specimens).

It should be noted that among suppliers there is no consensus on the method of obtaining the interferent (hemolyzate/Hb overload), on the choice of wavelengths (monochromatism, bichromatism or multichromatism), on the algorithm of calculation of the index and on the mode of expression of the importance of hemolysis (quantitative or semi-quantitative). As a result, the allowable HI cut-offs determined in our study on Cobas 8000 are not transferable to another. The HI measurements are homogeneous for a couple of identical analyzers but vary from one type of analyzer to another.

Overall, our results are of particular interest in improving the management of laboratory quality results and optimizing patient care with appropriate resampling. The European Federation of Clinical Chemistry and Laboratory Medicine states the practical recommendations for the management of test results in hemolyzed samples [10, 26]. Regarding the definition of HI cut-offs, Lippi et al. reported that ACL-based HI cut-off could be used for the purpose of annotating results indicating the direction of the variation, while both TCL- or RCV-based HI cut-off could be used to identify clinically significant interference that needs a cancellation of results [11]. The use of two cut-offs such as analytical and clinical cut-offs (RCV) allows a gradation of the interference to hemolysis, which could help clinicians: firstly, no interference when $\mathrm{HI}$ is below $\mathrm{CV}_{\mathrm{a}}$, "alert index" when $\mathrm{HI}$ comprises between $\mathrm{CV}_{\mathrm{a}}$ and RCV, and an unacceptable index when $\mathrm{HI}$ is above RCV. In our study, only one cut-off was discussed, and the TCL considered as clinical cut-off appeared to be the most adapted. The use of one cut-off taking into account the analytical and biological variation is a good compromise and easy to implement in the laboratory information system. In addition, since we have tested hemolysis interference on several levels of analyte value, we can also establish a gradation in the management of hemolysis interference. For example, it is not necessary for a patient with an iron $>30 \mu \mathrm{mol} / \mathrm{L}$ to request another sample if the $\mathrm{HI}$ is $<5.1 \mathrm{~g} / \mathrm{L}$, while for an iron $<30 \mu \mathrm{mol} / \mathrm{L}$, the allowable $\mathrm{HI}$ is $2.5 \mathrm{~g} / \mathrm{L}$. All troponin results should be deleted from the report in the presence of a $\mathrm{HI}$ of $2.5 \mathrm{~g} / \mathrm{L}$. However, following our results, for a troponin $<20 \mathrm{ng} / \mathrm{mL}$, with a HI between 1.4 and $1.8 \mathrm{~g} / \mathrm{L}$, a comment specifying an underestimation of 10\%-15\% may be added below the result, and for the troponin results between 20 and $60 \mathrm{ng} / \mathrm{mL}$, the results must be deleted from the report when $\mathrm{HI}$ is $>1.8 \mathrm{~g} / \mathrm{L}$.

Based on our data, the use of $10 \% \Delta$ as a significant bias seems to be too general, the ACL is too restrictive and could be used to alert about the presence of hemolysis, and the RCV, if used alone, is not suitable to certain analytes such as hs-cTnT. To standardize the management of hemolysis interference for the analytes studied, our study confirms that the TCL is the most appropriate calculation method in practice for biochemistry and immunochemical parameters. Also, it is strongly advised on the other hand 
to test the interference criteria on at least two values of analytes as suggested by Von Meyer et al. [26]. Following our results, the conditions for reporting results for several parameters will be broadened, particularly in the case of pathological values avoiding resampling.

\section{Conclusions}

In conclusion, the use of TCL-based HI cut-off would best serve the expectations of clinicians and the best compromise for making the decision to suppress the analyte result on the laboratory report when $\mathrm{HI}$ values measured on the samples exceed it. In addition, our results highlight different maximum allowable $\mathrm{HI}$ according to analyte value using Cobas 8000@ from Roche Diagnostics. This own determination of HI appears essential to ensure patient safety and to avoid overestimating hemolysis and re-sampling resulting in delayed patient care. However, implementation in practice in each lab is difficult. Recently, the European Federation of Clinical Chemistry and Laboratory Medicine carried out a survey on the management by laboratories of hemolytic, icteric and lipemic samples, highlighting a fairly significant heterogeneity [27]. As a result, a general consensus in the field regarding comments or cancellation of the test results affected by hemolysis is expected.

Acknowledgments: We gratefully acknowledge all the laboratory technicians for their assistance.

Author contributions: All the authors have accepted responsibility for the entire content of this submitted manuscript and approved submission.

Research funding: None declared.

Employment or leadership: None declared.

Honorarium: None declared.

Competing interests: The funding organization(s) played no role in the study design; in the collection, analysis, and interpretation of data; in the writing of the report; or in the decision to submit the report for publication.

\section{References}

1. Lippi G, Blanckaert N, Bonini P, Green S, Kitchen S, Palicka V, et al. Haemolysis: an overview of the leading cause of unsuitable specimens in clinical laboratories. Clin Chem Lab Med 2008;46:764-72.

2. Heiligers-Duckers C, Peters NA, van Dijck JJ, Hoeijmakers JM, Janssen MJ. Low vacuum and discard tubes reduce hemolysis in samples drawn from intravenous catheters. Clin Biochem 2013;46:1142-4.
3. Heyer NJ, Derzon JH, Winges L, Shaw C, Mass D, Snyder SR, et al. Effectiveness of practices to reduce blood sample hemolysis in EDs: a laboratory medicine best practices systematic review and meta-analysis. Clin Biochem 2012;45:1012-32.

4. Liu D, Li Y, Huang Y. The prevalence of hemolysis - a survey using hemolysis index. Clin Chem Lab Med 2017;55:e90-1.

5. Mrazek C, Simundic AM, Wiedemann H, Krahmer F, Felder TK, Kipman $U$, et al. The relationship between vacuum and hemolysis during catheter blood collection: a retrospective analysis of six large cohorts. Clin Chem Lab Med 2017;55:1129-34.

6. Ryder KW, Trundle DS, Bode MA, Cole RE, Moorehead WR, Glick MR. Effects of hemolysis, icterus, and lipemia on automated immunoassays. Clin Chem 1991;37:1134-5.

7. Ali D, Sacchetto E, Dumontet E, Le Carrer D, Orsonneau JL, Delaroche $\mathrm{O}$, et al. Hemolysis influence on twenty-two biochemical parameters measurement. Ann Biol Clin 2014;72:297-311.

8. CLSI. Interference testing in clinical chemistry; approved guideline-second edition. CLSI document EP07-A2. Wayne, PA: Clinical and Laboratory Standards Institute, 2015.

9. Farrell CJ, Carter AC. Hemolysis interference: are laboratories getting the information they need? Clin Chem 2016;62:1274-6.

10. Monneret D, Mestari F, Atlan G, Corlouer C, Ramani Z, Jaffre J, et al. Hemolysis indexes for biochemical tests and immunoassays on Roche analyzers: determination of allowable interference limits according to different calculation methods. Scand J Clin Lab Invest 2015;75:162-9.

11. Lippi G, Cadamuro J, von Meyer A, Simundic AM, European Federation of Clinical C, Laboratory Medicine Working Group for Preanalytical P. Practical recommendations for managing hemolyzed samples in clinical chemistry testing. Clin Chem Lab Med 2018;56:718-27.

12. Bastin P, Favresse J, Streel C, Maisin D, Fillée C, Gruson D. Assessment of in vitro stability: a call for harmonization across studies. Clin Chem Lab Med 2018;56:e121-4.

13. Oddoze C, Lombard E, Portugal H. Stability study of 81 analytes in human whole blood, in serum and in plasma. Clin Biochem 2012;45:464-9.

14. Ricós C, Alvarez V, Cava F, Garcia-Lario JV, Hernández A, Jimenez CV, et al. Current databases on biological variation: pros, cons and progress. Scand J Clin Lab Invest 1999;59: 491-500.

15. Barassi A, Pallotti F, Melzi d'Eril G. Biological variation of procalcitonin in healthy individuals. Clin Chem 2004;50:1878.

16. Nordenskjöld AM, Ahlström H, Eggers KM, Fröbert O, Jaffe AS, Venge $P$, et al. Short- and long-term individual variation in cardiac troponin in patients with stable coronary artery disease. Clin Chem 2013;59:401-9.

17. Ricós C, Cava F, García-Lario JV, Hernández A, Iglesias N, Jiménez $\mathrm{CV}$, et al. The reference change value: a proposal to interpret laboratory reports in serial testing based on biological variation. Scand J Clin Lab Invest 2004;64:175-84.

18. Ji JZ, Meng QH. Evaluation of the interference of hemoglobin, bilirubin, and lipids on Roche Cobas 6000 assays. Clin Chim Acta 2011;412:1550-3.

19. Agarwal S, Vargas G, Nordstrom C, Tam E, Buffone GJ, Devaraj S. Effect of interference from hemolysis, icterus and lipemia on routine pediatric clinical chemistry assays. Clin Chim Acta 2015;438:241-5. 
20. Dolci A, Panteghini M. Harmonization of automated hemolysis index assessment and use: is it possible? Clin Chim Acta 2014;432:38-43.

21. Roffi M, Patrono C, Collet JP, Mueller C, Valgimigli M, Andreotti F, et al. 2015 ESC Guidelines for the management of acute coronary syndromes in patients presenting without persistent ST-segment elevation: Task Force for the Management of Acute Coronary Syndromes in Patients Presenting without Persistent ST-Segment Elevation of the European Society of Cardiology (ESC). Eur Heart J 2016;37:267-315.

22. Florkowski C, Wallace J, Walmsley T, George P. The effect of hemolysis on current troponin assays - a confounding preanalytical variable? Clin Chem 2010;56:1195-7.

23. Snyder JA, Rogers MW, King MS, Phillips JC, Chapman JF, Hammett-Stabler CA. The impact of hemolysis on Ortho-Clinical Diagnostic's ECi and Roche's elecsys immunoassay systems. Clin Chim Acta 2004;348:181-7.

24. Dimeski G. Effects of hemolysis on the Roche ammonia method for Hitachi analyzers. Clin Chem 2004;50:976-7.

25. Perović A, Dolčić $M$. Influence of hemolysis on clinical chemistr! parameters determined with Beckman Coulter tests - detection of clinically significant interference. Scand J Clin Lab Invest 2019;79:154-9.

26. Von Meyer A, Cadamuro J, Lippi G, Simundic AM. Call for more transparency in manufacturers declarations on serum indices: on behalf of the Working Group for Preanalytical Phase (WG-PRE), European Federation of Clinical Chemistry and Laboratory Medicine (EFLM). Clin Chim Acta 2018;484:328-32.

27. Cadamuro J, Lippi G, von Meyer A, Ibarz M, van Dongen-Lases $E$, Cornes $M$, et al. European survey on preanalytical sample handling - part 2: practices of European laboratories on monitoring and processing haemolytic, icteric and lipemic samples. On behalf of the European Federation of Clinical Chemistry and Laboratory Medicine (EFLM) Working Group for the Preanalytical Phase (WG-PRE). Biochem Med (Zagreb) 2019;29:020705. 